\title{
MODELAMIENTO FÍSICO A ESCALA DE PILOTES INDIVIDUALES, DE CABEZA LIBRE, SOMETIDOS A CARGA LATERAL EN SUELOS COHESIVOS
}

\author{
Physical scale modeling of single free head piles under lateral loading in cohesive soils \\ Edgar Leonardo Salamanca-Medina ${ }^{1}$, Nebardo Arturo Abril-González ${ }^{2}$ \\ ${ }^{1}$ Esp., Universidad Pedagógica y Tecnológica de Colombia (Sogamoso, Boyacá, Colombia). \\ edgarleonardo.salamanca@gmail.com. \\ ${ }^{2}$ M.Sc., Universidad Pedagógica y Tecnológica de Colombia (Sogamoso, Boyacá, Colombia). \\ nebardo.abril@uptc.edu.co.
}

(Recibido abril 24 de 2017 y Aprobado junio 2 de 2017)

\begin{abstract}
Resumen
Este artículo presenta los resultados de la modelación a pequeña escala de pilotes de madera, de cabeza libre, sometidos a carga horizontal en suelos cohesivos, ensayados con el fin de comparar los resultados con modelos analíticos propuestos por varios autores. Se emplearon los métodos de la carga característica (CLM) y curvas P-Y, para la predicción de las deflexiones laterales en la cabeza de los pilotes, y el método propuesto por Broms para la estimación de resistencia por carga última lateral. Estas predicciones se compararon con los resultados del modelamiento físico, y se obtuvo bastante aproximación entre ellos.
\end{abstract}

Palabras clave: pilotes; deflexión lateral; geotecnia; arcilla; modelos.

\begin{abstract}
This paper presents the results of the small scale modeling of free head wood piles under horizontal loading in cohesive soils, tested in order to compare the results with analytical models proposed by various authors. Characteristic Load (CLM) and P-Y Curves methods were used for the prediction of lateral deflections at the head of the piles and the method proposed by Broms for estimating the ultimate lateral load. These predictions were compared with the results of the physical modeling, obtaining a good approximation between them.
\end{abstract}

Keywords: piles; lateral deflection; Geotechnics; clay; models.

\section{INTRODUCCIÓN}

Los sistemas de cimentación con pilotes se utilizan comúnmente en situaciones donde la respuesta de los estratos superficiales no garantiza la estabilidad de las estructuras emplazadas sobre ellos.

Por lo general, las cimentaciones tienen por función transmitir al terreno de la forma más adecuada, cargas verticales, producto de la carga viva y carga muerta de las estructuras, aunque en situaciones particulares, adicionalmente experimentan esfuerzos horizontales, como resultado de empujes de tierras sobre estructuras de contención, corrientes de río sobre pilas de puentes, cargas sísmicas en edificios y puentes, cargas por oleaje en estructuras offshore, cargas de tensión en cables en torres de transmisión eléctricas, aceleración y frenado de vehículos en puentes, entre muchas otras [1].

Sistemas de pilotes cargados horizontalmente también pueden aplicarse como contención en fenómenos de remoción en masa, tan comunes en nuestro medio, dados los fuertes periodos invernales asociados al fenómeno de La Niña [2].

Algunos códigos de construcción especifican que los pilotes se diseñen de manera tal que la estructura pueda resistir una fuerza lateral del $10 \%$ de la fuerza aplicada axialmente. En el diseño de algunas fundaciones de pilotes, existen dos criterios para satisfacer. El primero, 
un adecuado factor de seguridad antes de la falla última, y el segundo, aceptables deformaciones ante la carga solicitada. Este estudio se centró tanto en la deflexión lateral como en la carga lateral última de pilotes a escala reducida en suelos cohesivos cuando se cargan horizontalmente. La motivación para este trabajo fue el deseo de contrastar los resultados teóricos de los métodos considerados, con los obtenidos en las mediciones del modelo físico. Esto puede significar un primer paso en el camino de establecer el grado de confianza que pueda tenerse en nuestro medio cuando se aplique alguna de las metodologías propuestas en el pasado por los investigadores.

\section{ASPECTOS TEÓRICOS}

El diseño de pilotes sometidos a cargas horizontales puede estar en función de la carga máxima lateral que estos puedan soportar, o en función del máximo desplazamiento lateral que se presente. El desplazamiento admisible puede ser relativamente alto en estructuras no permanentes o en muros de contención, pero en puentes o estructuras esbeltas es limitado a valores pequeños.

Según la ubicación de sus cargas laterales, los pilotes se clasifican en pilotes activos y pilotes pasivos. Los primeros son aquellos sometidos a cargas aplicadas en la cabeza, como es el caso de las cimentaciones de plataformas petrolíferas, muros de contención y pilares de viaductos. Los pilotes pasivos soportan cargas a lo largo de su longitud y se emplean como estructuras de contención de inestabilidades, ya sea como cuerpo de las pantallas o formando filas de elementos muy juntos (pantallas de pasadores), también se usan en estribos de puentes con taludes en terraplén. Las metodologías seguidas para evaluar el comportamiento de pilotes sometidos a carga horizontal van desde análisis simples de carga última, análisis lineales de deflexiones y esfuerzos, procedimientos manuales simplificados basados en análisis no lineales, hasta análisis iterativos basados en resortes no lineales [3]; estos últimos han tenido buena aceptación en el medio científico, pues sus resultados modelan con gran exactitud el comportamiento no lineal de la interacción suelo (o roca)-pilote, bajo cargas laterales, aunque su aplicación requiere de programas computacionales. La bibliografía expone que el valor de los desplazamientos es bastante sensible a los parámetros del suelo en los niveles más superficiales. Se ha en- contrado que solo el suelo dentro de los ocho diámetros de pilote desde la superficie del terreno es importante para las condiciones de cargas laterales, debido a que casi toda la carga lateral se transfiere de los pilotes activos en el suelo dentro de esta longitud [4]. Por ello, es conveniente determinar de manera exacta los parámetros geotécnicos en esta profundidad crítica. Los métodos usados en este estudio para pilotes activos se tratan a continuación.

\subsection{Método de Broms}

Para desarrollar su método de cálculo de la resistencia última del suelo $Q u$, Broms introdujo unas simplificaciones a la forma como se distribuyen las presiones de reacción del suelo a lo largo del pilote, según las condiciones presentes en la cabeza del pilote: pilotes no restringidos (de cabeza libre) y pilotes restringidos (de cabeza fija o cabeza empotrada). Además, consideró de manera separada suelos friccionantes y suelos cohesivos, aplicando el concepto de pilotes cortos y pilotes largos. Los primeros, dependientes de la resistencia del suelo, y los últimos, del momento de fluencia My del elemento estructural, llegando a las siguientes expresiones:

$$
\begin{aligned}
& M_{\max }=Q u(e+1.5 D+0.5 f) \\
& f=\frac{Q u}{9 C u D} \\
& M y=\sigma_{E}\left(\frac{\pi D^{3}}{32}\right)
\end{aligned}
$$

Donde $Q u$ es la carga última lateral, $C u$ es la cohesión no drenada, My el momento de fluencia del pilote para una sección circular sólida, $D$ es el diámetro del pilote, $L$ la longitud del pilote por debajo de la superficie, $e$ es la distancia de aplicación de la carga medida desde la superficie del suelo y $\sigma_{E}$ la resistencia a la compresión para pilotes en concreto o el limite elástico para pilotes de madera o acero.

El procedimiento para obtener la carga última Qu para pilotes de cabeza libre en suelos cohesivos se resume a continuación:

* Obtener Qu de la Figura 1.

*Cálculo de Mmáx utilizando simultáneamente las ecuaciones 1 y 2.

*Cálculo de My para la sección del pilote: 
Si My > Mmáx, Qu es la carga última lateral (pilote corto).

Si Mmáx > My, hallar Qu a partir de My en la Figura 2 (pilote largo).

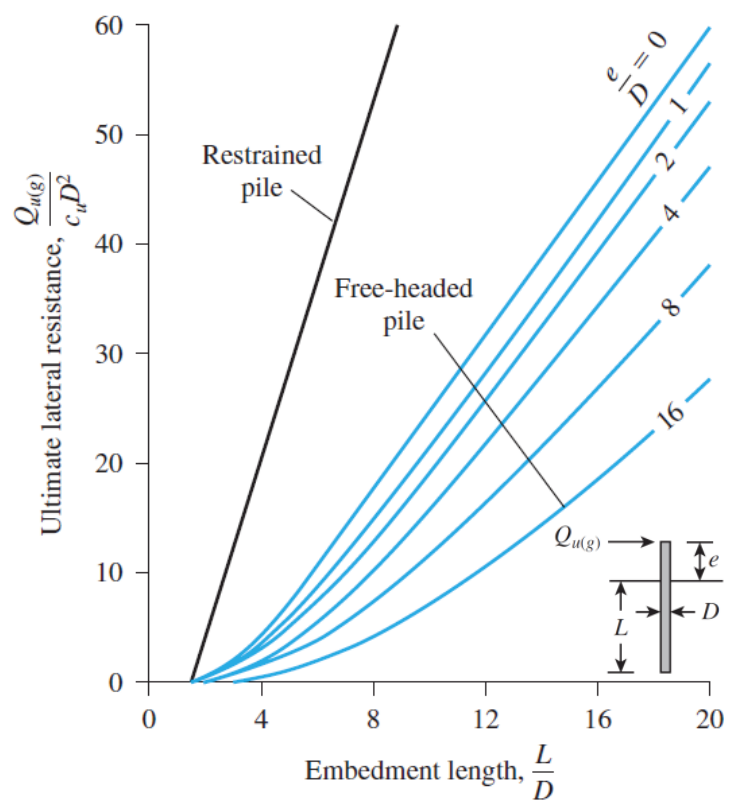

Figura 1. Solución de Broms para la resistencia última lateral de pilotes cortos en suelos cohesivos [5].

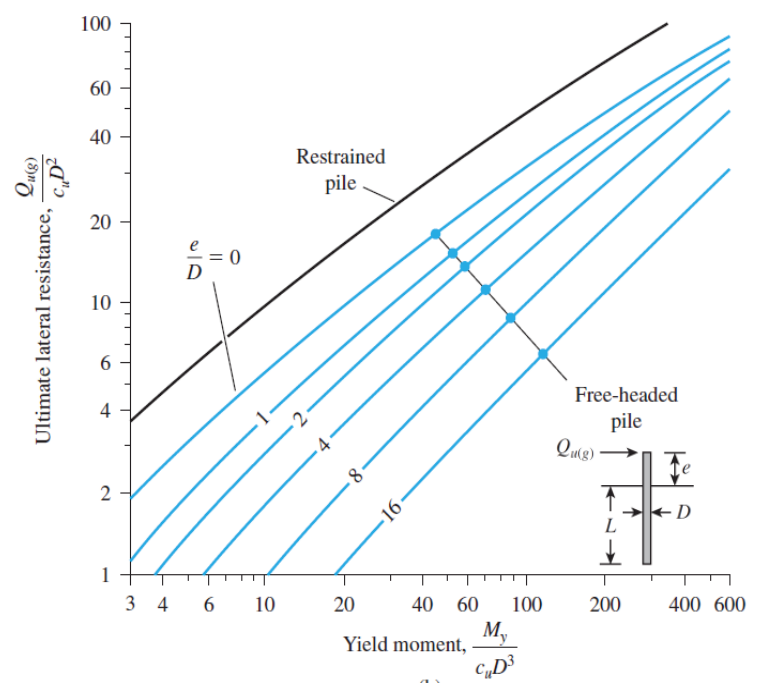

Figura 2. Solución de Broms para la resistencia última lateral de pilotes largos en suelos cohesivos [5].

Las deflexiones laterales para cargas de trabajo mediante este método pueden ser calculadas solo si la carga de trabajo $Q g$ está entre 0.3 y 0.5 veces la carga ultima
$Q u$ [6]. La Figura 3 da las relaciones entre la cantidad adimensional $\beta^{*} \mathrm{~L}$ y $\left(\mathrm{X}_{\mathrm{z}} \mathrm{KDL}\right) / \mathrm{Qg}$ tanto para los pilotes de cabeza libre como para pilotes de cabeza empotrada o restringida:

$$
\beta=\sqrt[4]{\frac{K D}{4 E p I p}}
$$

Donde $E p$ es el módulo de elasticidad del pilote, I $p$ el momento de inercia para determinada sección del pilote, $K$ es el coeficiente de reacción horizontal del suelo (módulo de balasto), $D$ diámetro del pilote, $X_{z}$ la deflexión lateral a nivel del suelo y $L$ es la longitud del pilote por debajo de la superficie.

Las siguientes condiciones expresan otra forma para determinar si un pilote de cabeza libre o cabeza empotrada se considera largo o corto en suelos arcillosos [7]:

Pilote largo cuando $\beta L>2.25$

Pilote corto cuando $\beta \mathrm{L}<2.25$

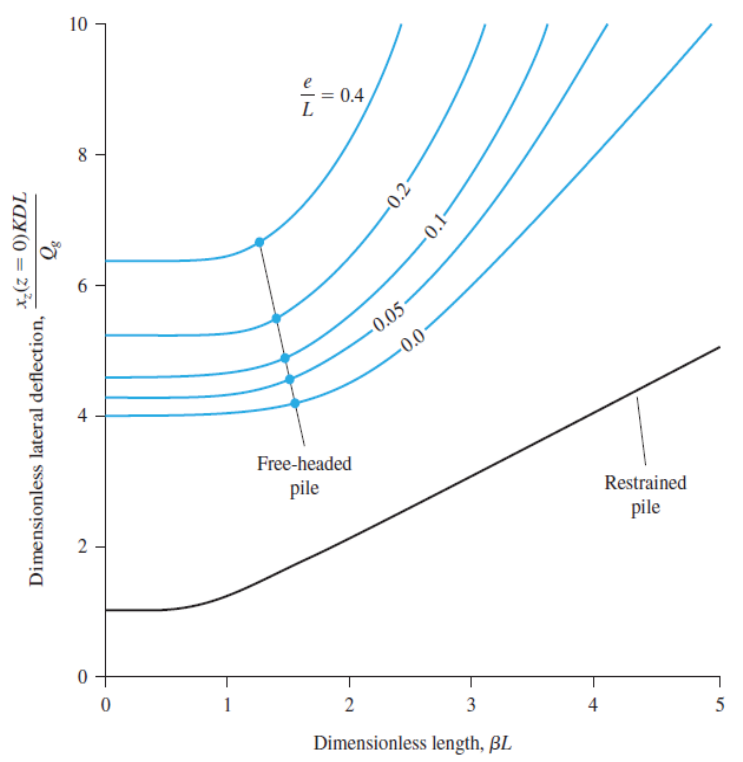

Figura 3. Cálculo de la deflexión lateral lineal en pilotes para cargas horizontales de trabajo en suelos cohesivos según Broms [5].

En suelos cohesivos, el módulo de reacción horizontal o módulo de balasto se supone aproximadamente constante con la profundidad [8]. Vesic [9] propuso la siguiente ecuación para estimar el valor de $K$ : 


$$
K=\left(\frac{0.65}{D}\right)\left(\sqrt[12]{\frac{E s D^{4}}{E p I p}}\right)\left(\frac{E s}{1-v^{2}}\right)
$$

Con Es módulo de elasticidad del suelo y u módulo de Poisson del suelo. Otra relación empírica para el cálculo del valor de $K$ para suelos cohesivos es formulada por Davisson [10], un poco más conservadora:

$$
K=67\left(\frac{C u}{D}\right)
$$

\subsection{Método de la carga característica (CLM)}

El método de la carga característica (CLM) fue desarrollado por Duncan et al., considerando una serie de soluciones no lineales de curvas p-y para un rango de condiciones en la cabeza del pilote y para un rango de suelos. Estos resultados fueron analizados como variante simplificada del método de las curvas P-Y, para obtener ecuaciones simples que permitan definir el comportamiento de los pilotes bajo carga lateral. Este método es muy útil y práctico para determinar las deflexiones en la cabeza del pilote producto de cargas laterales bajo condiciones de cabeza libre y cabeza empotrada, tratando de manera independiente suelos granulares y suelos cohesivos. Para llegar a las predicciones, es preciso determinar la fuerza lateral característica $P c$, que en suelos arcillosos homogéneos es:

$$
P c=7.34 D^{2}(E p R i)\left(\frac{C u}{E p R i}\right)^{0.68}
$$

Donde $D$ es el diámetro del pilote, $E p$ corresponde al módulo de elasticidad del pilote, $\mathrm{Cu}$ es la cohesión no drenada del suelo y $R i$ es la relación del momento de inercia entre la sección del pilote interna con la sección externa. Evans propuso caracterizar el comportamiento no lineal de los pilotes a través de las relaciones entre variables adimensionales. Brettmann y Duncan interpolaron los resultados numéricos para la relación $P-y$ como:

$$
\frac{y}{D}=a_{h}\left(\frac{P}{P_{c}}\right)^{B h}
$$

Donde $y$ corresponde a la deflexión lateral en la cabeza del pilote, $D$ al diámetro del pilote, $P c$ a la carga característica (obtenida mediante la ecuación 7), $a h, \mathrm{~B} h$, constantes dependientes de la condición en la cabeza del pilote y $P$ es la carga de trabajo arbitraria según el diseño. Las constantes se dan en la Tabla 1 en función del mecanismo de fijación en la cabeza del pilote para un suelo arcilloso. Estas constantes, dadas por Brettman y Duncan, han sido confirmadas sustancialmente por retroanálisis.

Tabla 1. Valores constantes para suelos arcillosos por el método de la carga característica [11]

\begin{tabular}{ccc}
\hline Constante & Cabeza libre & Cabeza empotrada \\
\hline$a_{h}$ & 50 & 14 \\
$B_{h}$ & 1.822 & 1.846 \\
\hline
\end{tabular}

El CLM es un instrumento útil y práctico, que permite predecir los efectos no lineales carga-deflexión sin una caracterización compleja de las características de deformación no lineal del suelo. De hecho, el único parámetro de suelo necesario es el parámetro de resistencia $\mathrm{Cu}$ para arcillas.

\subsection{Método de las curvas $P-Y$}

Para una evaluación rigurosa de la respuesta de pilotes sometidos a cargas laterales, es necesario llegar a la solución de una ecuación diferencial no lineal, que represente el comportamiento no lineal del suelo y del pilote. Obviamente es necesario para ello, plantear expresiones no lineales en las que la resistencia y la elasticidad del suelo estén en función del pilote a lo largo de este. Esto, en principio, representa una dificultad, pero con el empleo de los métodos numéricos se llega a soluciones bastante razonables y que concuerdan en alto grado con los resultados de los modelos experimentales. El método de las curvas P-Y fue propuesto a finales de 1940 y ha tenido significativas contribuciones hasta la fecha, dada su importancia en el análisis de plataformas petroleras mar adentro, que deben diseñarse para resistir el efecto de las cargas producidas por el oleaje, los sismos y los vientos. El desarrollo de las ecuaciones diferenciales se debe a Timoshenko y otros autores; la ecuación no lineal del problema se expresa de la forma siguiente:

$$
E p I p \frac{\partial^{4} y}{\partial x^{2}}+\frac{\partial^{2} y}{\partial x^{2}}+E_{P Y} y=0
$$


En esta ecuación, $x$ es la distancia medida desde cabeza del pilote, $y$ es la deflexión, Eplp es la rigidez a flexión y Epy corresponde al módulo de reacción del pilote bajo carga horizontal. Pertenece a una ecuación diferencial no lineal, pues el valor de Epy es una función de la profundidad $x$ considerada, $y$ de la deflexión $y$ del pilote en ese punto. No es pertinente incluir en este artículo la deducción de la ecuación diferencial.

Para obtener las curvas p-y tipo, correspondientes a suelos arcillosos con diferentes condiciones de agua freática y distinta consistencia, varios autores desarrollaron las soluciones a la ecuación diferencial no lineal para tres modelos; también se han desarrollado curvas P-Y tipo para suelos granulares, suelos cohesivos-friccionantes y rocas, que no serán tratadas aquí. La obtención de los modelos se sustenta en los resultados de pruebas instrumentadas de pilotes sometidos a carga lateral, para lo cual fue necesario realizar la caracterización del terreno, incluyendo la rigurosa definición de sus parámetros de resistencia y rigidez. Así mismo, se determinaron las dimensiones y rigideces de los pilotes de manera muy precisa. Los modelos para suelos arcillosos son los siguientes [12]:

Arcilla blanda con presencia del nivel freático. Arcilla dura sin nivel freático presente.

Arcilla dura con presencia de nivel freático.

Los parámetros necesarios para aplicar cada uno de los modelos anteriores son la cohesión no drenada $\mathrm{Cu}$, la deformación unitaria para el $50 \%$ de la resistencia a la compresión de la arcilla $\varepsilon 50$, y la rigidez inicial de la arcilla $K_{p y}$ (solo para arcillas duras), parámetros que son obtenidas a partir de ensayos de laboratorio y/o correlaciones con $\mathrm{Cu}$ [12-13].

Algunas soluciones numéricas, incluidas en ciertos programas de modelamiento geotécnico, contienen las curvas p-y tipo para diferentes tipos de materiales. Pueden citarse el LPILE implementado por la compañía Enfsoft.inc y el RSPile de Rocsience. Dichos programas son algo costosos y pocas instituciones académicas en el país los poseen. Las predicciones por el método de las curvas P-Y para este trabajo se realizaron utilizando el software libre PyPile V.0.2.2, investigado por la compañía Yong Technology Inc. y adquirido por los autores mediante consultas técnicas. Este software es de uso limitado, que difiere de los anteriormente citados en opciones de configuración geométrica del terreno, análisis de grupos de pilotes, aplicación de cargas axiales y laterales simultáneamente, variedad de curvas $p$-y tipo, entre otras.

\section{PROCEDIMIENTO EXPERIMENTAL}

El desarrollo experimental consistió en someter a carga lateral, pilotes individuales, de madera, de cabeza libre con dimensiones a escala, que se instalaron en un suelo arcilloso previamente dispuesto dentro de un contenedor de vidrio con dimensiones $28.5 \mathrm{~cm} \times 28.5 \mathrm{~cm} \times 90$ $\mathrm{cm}$. El montaje se muestra esquemáticamente en la Figura 4 y con imágenes reales en las Figuras 5 y 6 .

El recipiente contenedor se llenó de arcilla remoldeada en capas compactadas, variando para cada una de las pruebas, tanto la humedad como el espesor de las capas y la energía de compactación, a fin de tener cada vez diferentes propiedades físicas y mecánicas (ver Tabla 3 ). El parámetro Cu se obtuvo con penetrómetro de bolsillo, en lo posible dentro de los ocho diámetros de pilote por debajo de la superficie. Otros parámetros de resistencia se tomaron de trabajos anteriores. Se ensayaron 5 pilotes de madera (eucalipto) con diámetro de $2.3 \mathrm{~cm}$ y longitudes comprendidas entre $39 \mathrm{~cm}$ y $61 \mathrm{~cm}$, como se detalla en la Tabla 5. El hincado se efectuó inicialmente en cada caso con presión y luego con golpes de martillo de mano, cuidando de no perder la verticalidad; después se ató un cable a la cabeza del pilote, el cual terminaba en una base metálica, sobre la cual se colocaron las pesas que tensionaban el cable transmitiendo la carga horizontal. Se instaló un deformímetro contra la cabeza del pilote, para registrar el desplazamiento horizontal.

Tabla 2. Parámetros de rigidez de los pilotes [14]

\begin{tabular}{ccc}
\hline $\begin{array}{c}E p \\
(\mathrm{KPa})\end{array}$ & $\boldsymbol{v}$ & $\begin{array}{c}\text { Limite elástico } \boldsymbol{\sigma}_{\mathrm{E}} \\
(\mathrm{kPa})\end{array}$ \\
9000000 & 0.3 & 15000 \\
\hline
\end{tabular}




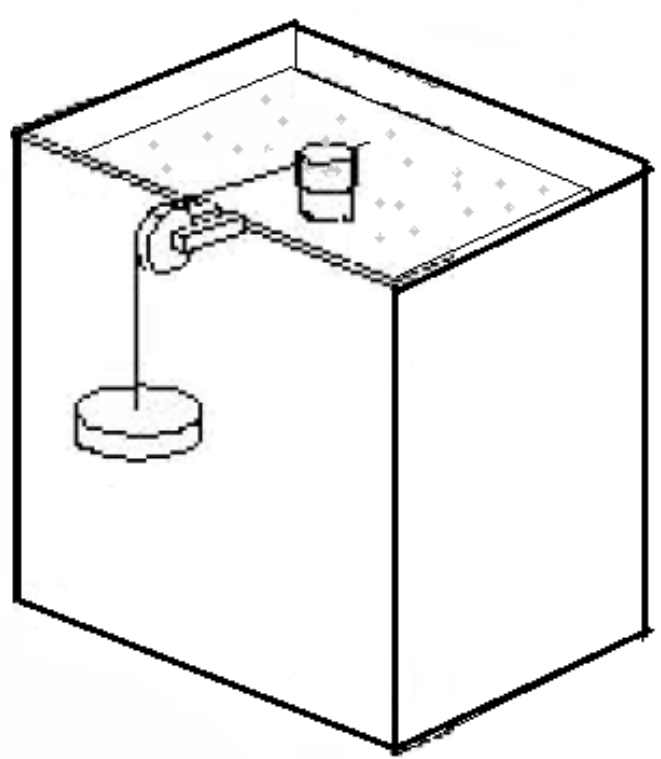

Figura 4. Configuración esquemática del aparato experimental.

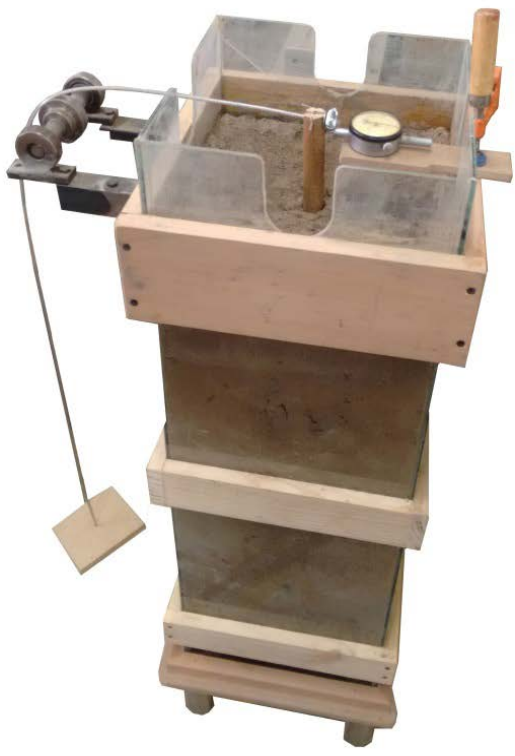

Figura 5. Montaje final del pilote, aparatos de medición y carga en el recipiente contenedor.
Tabla 3. Propiedades geotécnicas de la arcilla

\begin{tabular}{|c|c|c|c|c|c|}
\hline & $\begin{array}{c}\text { Humedad } \\
\text { natural } \\
(\%)\end{array}$ & $\begin{array}{l}\mathrm{LL} \\
\text { (\%) }\end{array}$ & $\begin{array}{l}\text { IP } \\
\text { (\%) }\end{array}$ & $\begin{array}{l}\text { Peso } \\
\text { unitario } \\
\left(\mathrm{kN} / \mathrm{m}^{3}\right)\end{array}$ & $\begin{array}{c}\mathrm{Cu} \\
(\mathrm{kPa})\end{array}$ \\
\hline 1 & 33 & 51 & 26 & 17.6 & 25 \\
\hline 2 & 36 & 51 & 26 & 17.6 & 25 \\
\hline 3 & 34 & 51 & 26 & 17.6 & 25 \\
\hline 4 & 29 & 51 & 26 & 18.5 & 30 \\
\hline 5 & 28 & 51 & 26 & 18.5 & 30 \\
\hline \\
\hline & $\begin{array}{c}\text { Es } \\
(\mathrm{kPa})\end{array}$ & & $v$ & \multicolumn{2}{|c|}{$\varepsilon 50$} \\
\hline & 350 & & 0.4 & \multicolumn{2}{|c|}{0.02} \\
\hline
\end{tabular}

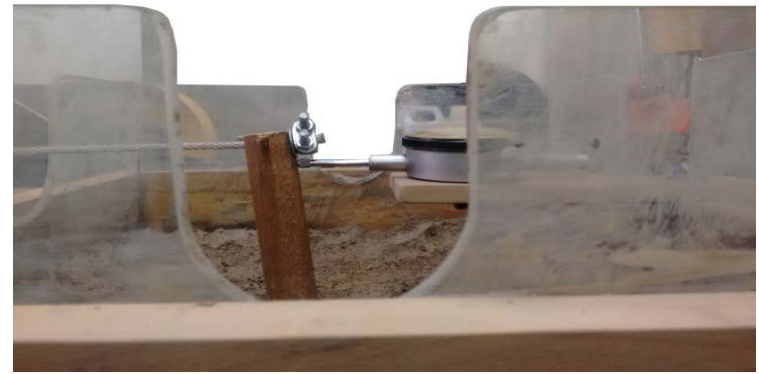

Figura 6. Deflexión lateral en uno de los pilotes ensayados.

\section{RESULTADOS Y COMPARACIÓN}

Los resultados de la modelación física y de la aplicación de los métodos considerados se presentan en las Tablas 5 y 6; también se muestran en la Figura 7 las curvas p-y para los cinco pilotes, comparando las deflexiones laterales por los métodos que predicen el comportamiento no lineal del suelo junto con la prueba de carga lateral experimental. La carga última se determinó analíticamente y se compararon para este valor las deflexiones últimas de carga, además del cálculo de deflexiones permisibles para cargas de trabajo. La carga de trabajo $Q g$ se normalizó como 0.4 veces la carga última obtenida. Los pilotes clasificaron como largos, y el valor de $K$ se determinó mediante la formulación de Vesic igual a $6494.76 \mathrm{kN} / \mathrm{m}^{3}$. 
La Figura 7 muestra la relación entre el desplazamiento y la carga horizontal lateral, observando un comportamiento marcadamente no lineal, incluso a niveles de fuerzas relativamente bajas. Además, los métodos predictivos, a simple vista, se ajustan en buena parte a la prueba de carga experimental, siendo muy marcada la proximidad de P-Y. Cabe aclarar que para el método de las curvas P-Y se seleccionó, a criterio de los autores, la curva p-y tipo arcilla blanda con presencia del nivel freático de acuerdo con las características físicas y mecánicas de la arcilla empleada.
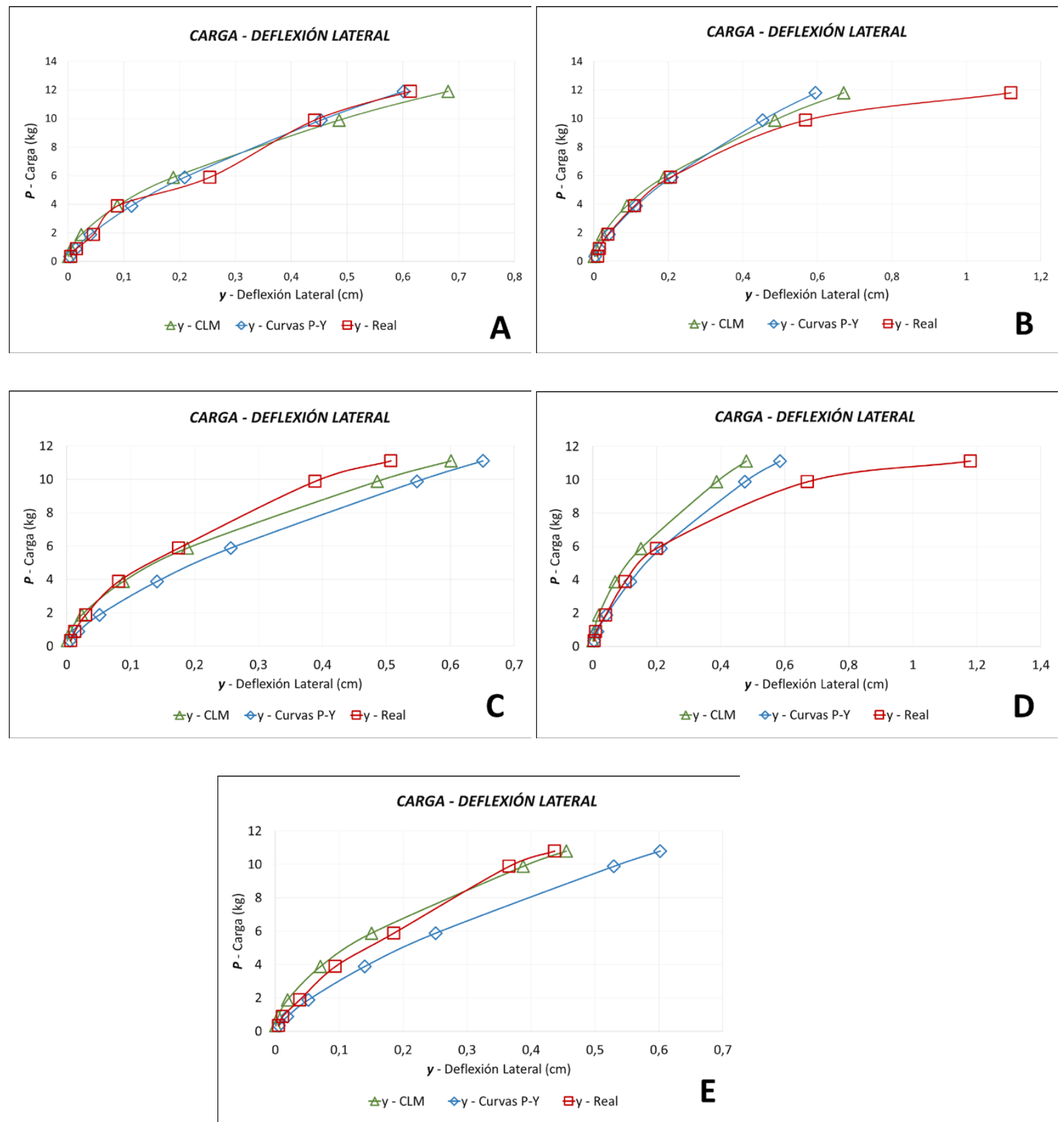

Figura 7. Carga vs. deflexión lateral y comparación de predicciones analíticas con los resultados reales. (A) Pilote 1. (B) Pilote 2. (C) Pilote 3. (D) Pilote 4. (E) Pilote 5. 
También se observa que las diferencias entre los resultados reales y las predicciones, por lo general, aumentan a medida que se incrementa el nivel de carga. Los resultados de carga última por el método de Broms demuestran que el modelo, en cuanto a la elección de los parámetros de rigidez del pilote, son acertados (calibración del modelo), que esta carga es inversamente proporcional a la longitud libre del pilote y sus resultados se acercan en gran parte a la realidad. En la Tabla 5 se observa que en las pruebas 2 y 4 las deflexiones reales no se ajustan con los métodos predictivos, debido a que la falla en el sistema se produce por un rápido y progresivo

Tabla 5. Comparación de las deflexiones laterales en la cabeza del pilote para las últimas cargas halladas

\begin{tabular}{|c|c|c|c|c|c|c|}
\hline \multirow{2}{*}{$\begin{array}{l}\text { Prueba } \\
\text { (pilote) }\end{array}$} & \multirow[t]{2}{*}{$\begin{array}{l}\text { Longitud pilote } \\
\qquad(\mathrm{cm})\end{array}$} & \multirow[t]{2}{*}{$\begin{array}{l}\text { Longitud libre } \\
\qquad(\mathrm{cm})\end{array}$} & \multirow{2}{*}{$\begin{array}{l}\text { Carga última } \\
\text { (Broms) } \\
\text { (kg) }\end{array}$} & \multicolumn{3}{|c|}{$\begin{array}{l}\text { Deflexiones según la carga última } \\
\qquad(\mathrm{cm})\end{array}$} \\
\hline & & & & Real & CLM & Curvas P-Y \\
\hline 1 & 61 & 10 & 11.9 & 0.614 & 0.681 & 0.601 \\
\hline 2 & 47 & 10 & 11.8 & 1.12 & 0.671 & 0.595 \\
\hline 3 & 54 & 11.5 & 11.109 & 0.507 & 0.601 & 0.651 \\
\hline 4 & 39 & 11.5 & 11.12 & 1.18 & 0.480 & 0.585 \\
\hline 5 & 50 & 12.5 & 10.8 & 0.437 & 0.455 & 0.602 \\
\hline
\end{tabular}

Tabla 6. Comparación de las deflexiones laterales en la cabeza del pilote para las cargas de trabajo

\begin{tabular}{ccccc|c|c|c}
\hline $\begin{array}{c}\text { Prueba } \\
\text { (pilote) }\end{array}$ & $\begin{array}{c}\text { Longitud } \\
\text { pilote } \\
\text { (cm) }\end{array}$ & \multirow{2}{*}{$\begin{array}{c}\text { Longitud libre } \\
\text { (cm) }\end{array}$} & & $\begin{array}{c}\text { Carga de } \\
\text { trabajo } \\
\text { (kg) }\end{array}$ & & \multicolumn{3}{c}{$\begin{array}{c}\text { Deflexiones según la carga de trabajo } \\
\text { (cm) }\end{array}$} \\
\cline { 5 - 8 } & & & & Real & CLM & Curvas P-Y & Broms \\
\hline 1 & 61 & 10 & 4.76 & 0.161 & 0.128 & 0.153 & 0.416 \\
2 & 47 & 10 & 4.72 & 0.149 & 0.126 & 0.151 & 0.502 \\
3 & 54 & 11.5 & 4.44 & 0.107 & 0.113 & 0.171 & 0.439 \\
4 & 39 & 11.5 & 4.45 & 0.129 & 0.091 & 0.142 & 0.667 \\
5 & 50 & 12.5 & 4.32 & 0.114 & 0.085 & 0.162 & 0.491 \\
\hline
\end{tabular}

desplazamiento lateral del pilote bajo una carga constante [15], y que en la condición experimental fueron de difícil control los rangos de cargas próximos a la falla, por el uso mismo de la disposición de las pesas; por tanto, esas lecturas fueron tomadas después de la falla. Es de resaltar que la observación de la falla en el modelo físico se encontró en el rango de cargas de $11 \mathrm{~kg}$ a los $13 \mathrm{~kg}$ para todas las pruebas y que su medición no fue precisa por lo mencionado anteriormente.

Ya que en la práctica los diseños se hacen para cargas de trabajo o cargas admisibles, se muestran en la Tabla 6 los resultados de deflexión para estas cargas calculadas teóricamente, junto con las deflexiones correspondientes en la modelación. Estas comparaciones observadas en la tabla mencionada muestran que el método de las curvas P-Y da una mejor correlación con los resultados reales, que los resultados obtenidos por el CLM, aunque se aclara que ambos métodos modelan la no linealidad del suelo y del pilote. Las predicciones dadas por el método de Broms son aceptables, pero distantes de los resultados experimentales, tal vez por tratarse de un método que modela linealmente el suelo y el pilote bajo carga lateral.

En todo caso, más allá de las diferencias encontradas, el método de Broms resulta de sustancial importancia para determinar los valores de las cargas máximas que 
pueden aplicarse a pilotes sometidos a carga lateral, con un criterio conservador de deflexiones permisibles.

Un análisis comparativo se hizo aplicando la expresión $\Sigma(\mathrm{Xi}-\mathrm{Xj})^{2}$ donde $i$ y $j$ son puntos correspondientes de la deflexión para una misma carga, tomando cada vez los resultados de dos procedimientos. Los resultados se presentan en la Tabla 7.

La prueba que presenta la mejor correlación de resultados es la 1 , dado que la sumatoria de las diferencias al cuadrado dio 0.003 para las curvas $\mathrm{P}-\mathrm{Y}$, y 0.0113 para el método CLM. También en la prueba 5 se manifiesta una aceptable correlación, pues los resultados para el mismo parámetro fueron 0.0606 para las curvas P-Y y 0.003 para el método CLM.

Tabla 7. Resultados de la comparación de suma de diferencias al cuadrado

\begin{tabular}{ccc}
\hline $\begin{array}{c}\text { Prueba } \\
\text { (pilote) }\end{array}$ & \multicolumn{2}{c}{$\Sigma(\mathbf{X i - X j )})^{2}$} \\
\cline { 2 - 3 } & Real Vs P-Y & Real Vs CLM \\
\hline 1 & 0.0030 & 0.0113 \\
2 & 0.2891 & 0.2097 \\
3 & 0.0567 & 0.0186 \\
4 & 0.3925 & 0.5741 \\
5 & 0.0606 & 0.0030 \\
\hline
\end{tabular}

Los resultados más dispersos corresponden a la prueba 4 , donde el parámetro evaluado fue de 0.3925 para las curvas P-Y y 0.5741 para el método CLM.

Vascones [16] propone el uso de un valor $R$ para evaluar la precisión de las mediciones, definido como la relación entre la deflexión medida y la deflexión de la predicción:

$$
R=\left(\frac{\text { Dmedida }}{\text { Dpredicción }}\right) *(100 \%)(10)
$$

Se concentraron los valores de $R$ para las deflexiones correspondientes a la carga admisible calculada para cada pilote. Los resultados se muestran en la Tabla 8. Un valor de $R$ de 0 a $100 \%$ es conservador, en cambio un valor de $R>100 \%$ es poco conservador.
Tabla 8. Valores de $R$ para los ensayos realizados

\begin{tabular}{cccc}
\hline \multirow{2}{*}{$\begin{array}{c}\text { Prueba } \\
\text { (Pilote) }\end{array}$} & $\begin{array}{c}\text { Carga de } \\
\text { trabajo } \\
(\mathbf{k g})\end{array}$ & $\begin{array}{c}\text { Real/P-Y } \\
\text { (\%) }\end{array}$ & $\begin{array}{c}\text { Real/CLM } \\
\text { (\%) }\end{array}$ \\
& & 105 & 126 \\
1 & 4.76 & 98 & 118 \\
2 & 4.72 & 63 & 95 \\
3 & 4.44 & 91 & 142 \\
4 & 4.45 & 70 & 134 \\
5 & 4.32 & &
\end{tabular}

Los valores de $R$ muestran gran cercanía con lo obtenido en la realidad en la pruebas 1 y 2 para $P-Y$ y en las pruebas 2 y 3 para CLM. Los valores más conservadores se obtienen por el método P-Y, mientras que solo en un caso CLM resultó ser conservador.

\section{CONCLUSIONES}

El diseño de pilotes sometidos a carga horizontal es un tema que involucra la predicción de deformaciones y la interacción suelo-estructura. Los resultados estarán en función de las características elásticas del pilote, de la naturaleza de las cargas aplicadas y de los parámetros del suelo. El método de las Curvas P-Y, por su buena correlación con los resultados experimentales, es una propuesta que considera esta interacción, y su empleo requiere el uso de aplicaciones computacionales. Para la comprensión de este problema, se ejecutó una experimentación física ejecutada por los autores bajo condiciones controladas, que incluyó el diseño, la fabricación y la construcción de elementos de fácil acceso a costos relativamente bajos. Este tipo de modelos físicos a escala ha cobrado importancia en el campo de la geotecnia, permitiendo conocer el comportamiento ante las solicitaciones que el proyecto tenga en su vida útil, propiciando óptimos diseños, bajo los criterios fundamentales en ingeniería: seguridad, funcionalidad y economía.

Aunque, de alguna forma, el factor escala puede estar influenciando los resultados, la comparación del comportamiento de los pilotes modelados experimentalmente con las predicciones de los análisis teóricos fue útil para identificar deficiencias y fortalezas de los métodos analíticos. 
Las predicciones analíticas para el cálculo de carga última por el método de Broms, aunque no pudieron ser medidas, resultaron ser bastante coherentes con lo visto físicamente, así que puede utilizarse para una estimación aproximada a la realidad de la carga de falla con fines de diseño. Las deflexiones laterales calculadas por los métodos no lineales (curvas P-Y, CLM), no coinciden en todos los casos con los resultados experimentales, pero sí se aproximan de manera notable en algunos de los modelos, lo cual indica que la modelación aplicada puede optimizarse para llegar a resultados plenamente aceptables.

La mejor correlación entre los resultados del modelo y el método P-Y se presenta en la prueba 1 , donde $\Sigma(\mathrm{Xi}$ $X \mathrm{Xj})^{2}=0.003$ y la mayor dispersión se tiene para la prueba 4 , con $\Sigma(X i-X j)^{2}=0.3925$.

La comparación entre los resultados del modelo y el método CLM, indican que la mejor correlación se obtuvo para la prueba 5 , con $\Sigma(X i-X j)^{2}=0.003$ y la mayor dispersión corresponde a la prueba 4 , donde $\Sigma\left(\mathrm{Xi}-\mathrm{Xj}^{2}\right)^{2}=$ 0.5741 .

Los resultados del método de las Curvas P-Y en este modelamiento resultaron ser los más conservadores para las cargas de trabajo calculadas en los cinco pilotes modelados.

Mediante la aplicación experimental de modelos a escala es posible verificar la validez de los métodos de diseño propuestos para pilotes sometidos a cargas laterales, desarrollados más allá de nuestras fronteras y que se apoyan tal vez en procedimientos similares. Lo anterior puede ser pretencioso, pero dado que los procesos de carga en pilotes involucran muchas variables, es válido contrastar resultados obtenidos en el laboratorio con aquellos que se determinan con las formulaciones matemáticas disponibles en la bibliografía actual.

\section{REFERENCIAS}

[1] INVÍAS, Manual de diseño de cimentaciones superficiales y profundas para carreteras. Bogotá: Ministerio de Transporte, 2012, pp. 7;42-7;66.

[2] N.A. Abril, E.G. Amaya y H.A. Fonseca, "Evaluación de amenazas por movimientos en masa causados en Jericó Boyacá, durante el periodo invernal de 2011", Revista Ingeniería Investigación y Desarro-
Ilo, vol. 16 n. ${ }^{\circ} 1$, pp. 6-13, enero, 2016. doi: https:// doi.org/10.19053/1900771X.5115

[3] R. Hernández, Análisis de pilotes cargados lateralmente, mediante interacción suelo estructura empleando una teoría simplificada de empujes. Bogotá: Universidad Nacional de Colombia, 2011.

[4] M. Duncan, Revision of the CLM Spreadsheet for Lateral Load Analyses of Deep Foundations. Virginia, EEUU: Center for Geotechnical Practice and Research, August, 2001, pp. 1-22.

[5] B. Das, Principles of Foundation Engineering, Eighth Edition. Boston, MA: Ed. Cengage Learning, 2016, pp. 456-470.

[6] J. Ruigrok, Laterally Load Piles: Models and Measurements. Holland: Delft, 2010.

[7] B. Broms, "Lateral Resistance of Piles in Cohesive Soils", Journal of the Soil Mechanics and Foundation Division, SM2, pp. 27-63, March, 1964.

[8] H. Poulus and E. Davis, Pile Foundation Analysis and Design. New York: Ed Wiley, 1974, pp. 143232.

[9] A. Vesic, "Design of Pile Foundations," presented in National Research Council, 1977, pp. 293-353.

[10] M.T. Davisson and H.L. Gill. "Laterally Loaded Piles in a Layered Soil System", Journal of Soil Mechanics and Foundation Division, ASCE, vol. 89 n. 3, pp. 63-94, 1963.

[11] C. Viggiani, Piles and Pile Foundations. UK: Taylor \& Francis, 2012, pp. 208-211.

[12] L. Reese and W. Van Impe, Single Piles and Pile Groups Under Lateral Loading. UK: Taylor \& Francis, 2001, pp. 70-84.

[13] P. Hannigan, Design and Construction of Driven Pile Foundations. USA: U.S. Department of Transportation, 1998, pp. 9;70-9;97.

[14] J. R. Sotomayor. (2003). Clasificación mecánica de la madera 100 especies mexicanas, presentado en XII Congreso Forestal Mundial, 2003 [online]. Disponible: http://www.fao.org/docrep/ARTICLE/ WFC/XII/1054-B4.HTM\#P14_128

[15] J.A. Ananthanathan, S. Gajan, T. Kanagalingam, H.N. Seneviratne. Behaviour of Laterally Loaded Piles. Sri Lanka: Department of Civil Engineering, University of Peradeniya, 2001.

[16] P. Vascones, Comportamiento de pilotes individuales bajo carga lateral: evaluación de un caso real. Guayaquil, Ecuador: Universidad Católica de Santiago de Guayaquil, 2010. 